ARTICLE

https://doi.org/10.1038/s41467-019-10757-1

\title{
Transcriptome and organellar sequencing highlights the complex origin and diversification of allotetraploid Brassica napus
}

Hong An (1) 1,2,8, Xinshuai Qi3 ${ }^{3,8}$, Michelle L. Gaynor (1) 4, Yue Hao (1) 5, Sarah C. Gebken ${ }^{1}$, Makenzie E. Mabry', Alex C. McAlvay ${ }^{6}$, Graham R. Teakle ${ }^{7}$, Gavin C. Conant ${ }^{5}$, Michael S. Barker (1) ${ }^{3}$, Tingdong Fu ${ }^{2}$, Bin $\mathrm{Yi}^{2} \&$ J. Chris Pires ${ }^{1}{ }^{1}$

Brassica napus, an allotetraploid crop, is hypothesized to be a hybrid from unknown varieties of Brassica rapa and Brassica oleracea. Despite the economic importance of $B$. napus, much is unresolved regarding its phylogenomic relationships, genetic structure, and diversification. Here we conduct a comprehensive study among diverse accessions from 183 B. napus (including rapeseed, rutabaga, and Siberian kale), 112 B. rapa, and 62 B. oleracea and its wild relatives. Using RNA-seq of $B$. napus accessions, we define the genetic diversity and subgenome variance of six genetic clusters. Nuclear and organellar phylogenies for B. napus and its progenitors reveal varying patterns of inheritance and post-formation introgression. We discern regions with signatures of selective sweeps and detect 8,187 differentially expressed genes with implications for $B$. napus diversification. This study highlights the complex origin and evolution of $B$. napus providing insights that can further facilitate $B$. napus breeding and germplasm preservation.

\footnotetext{
${ }^{1}$ Division of Biological Sciences, University of Missouri, Columbia, MO 65211, USA. ${ }^{2}$ National Key Lab of Crop Genetic Improvement, Huazhong Agricultura University, 430070 Wuhan, Hubei, P. R. China. ${ }^{3}$ Department of Ecology \& Evolutionary Biology, University of Arizona, Tucson, AZ 85721, USA. ${ }^{4}$ Department of Biology, University of Central Florida, Orlando, FL 32816, USA. ${ }^{5}$ Bioinformatics Research Center, North Carolina State University, Raleigh, NC 27695, USA. ${ }^{6}$ Department of Ecology and Evolutionary Biology, Cornell University, Ithaca, NY 14850, USA. 7 School of Life Sciences, University of Warwick, Coventry CV4 7AL, UK. ${ }^{8}$ These authors contributed equally: Hong An, Xinshuai Qi. Correspondence and requests for materials should be addressed to B.Y. (email: yibin@mail.hzau.edu.cn) or to J.C.P. (email: piresjc@missouri.edu)
} 
$B$ rassica napus is an allopolyploid species (AACC, $2 n=38$ ) thought to have formed by hybridization of two diploid species, Brassica rapa $(\mathrm{AA}=20)$ and Brassica oleracea $(C C=18)$, between 6800 and 12,500 years ago ${ }^{1-3}$. It is a versatile crop with global economic importance. There are three currently recognized subspecies: B. napus subsp. oleifera (rapeseed or rape), $B$. napus subsp. rapifera (rutabaga or swede), and B. napus subsp. pabularia (Siberian kale or leaf rape $)^{1,4}$. Rapeseed is a major vegetable oil resource, ranking second in worldwide oilseed production ${ }^{5}$. Cultivation of rapeseed started in Europe in the thirteenth century and became an important source of highquality lubricant oil during the industrial revolution ${ }^{6}$. In the past half-century, double-low (low erucic acid and low glucosinolate) rapeseed was produced by intensive breeding ${ }^{6,7}$. Today, rapeseed is also important for edible oil production and livestock forage. Rutabaga also has a long history, with the first printed record traced back to $1620^{8}$. Mainly cultivated in Europe and North America, rutabaga can be used both as a root vegetable and as fodder. The leafy vegetable morphotype Siberian kale is cultivated worldwide for human consumption ${ }^{9}$. Collectively, these crops are valued at more than 41 billion U.S. dollars for annual production $^{5}$.

Although the progenitors of $B$. napus are thought to be $B$. rapa and $B$. oleracea, the specific morphotypes that initially hybridized has yet to be identified. Currently, B. napus is believed to originate from the Mediterranean basin or some agricultural regions such as northern or western Europe, where B. rapa and B. oleracea co-exist ${ }^{10,11}$. Some molecular data, like RFLP and AFLP markers, indicate that $B$. napus was formed by several independent hybridization events ${ }^{12-14}$. However, whether the progenitors of $B$. napus were domesticated or wild is still unknown. According to several analyses of chloroplast and mitochondrial genomes, Brassica montana or other unknown Brassica species $(2 n=18)$ may also have contributed to the B. napus $\mathrm{C}$ genome as a maternal donor ${ }^{12,13,15}$. These Brassica species are also referred to as B. oleracea wild relatives or wild $C$ species as they have the same genomic structure as B. oleracea. However, there are also studies based on Brassica chloroplast genomes that have found that B. rapa is the maternal parent of B. napus, and furthermore, that $B$. rapa ssp. broccoletto (or 'Spring Broccoli Raab') is most closely related to the A genome ancestor of $B$. napus ${ }^{15,16}$. Many introgressions have been found both from $B$. rapa to $B$. napus and $B$. napus to $B$. rapa ${ }^{12,17}$, which further complicate the interpretations of these data.

Even though B. napus has a relatively short domestication history compared to $B$. rapa and some cereal crops like maize and Asian rice ${ }^{9,18}$, it has been domesticated into three distinct morphotypes: rapeseed, rutabaga, and Siberian kale. These types can be further clustered based on their growth habits into the winter types that need vernalization and the spring types that do not. $B$. napus can be also divided according to geographic region into European, North American, Asian, and Australian accessions. To date, almost all the studies on the diversity of B. napus crops have focused on the rapeseed subspecies. Of these studies, some find that the growth habit of $B$. napus better reflects its genetic structure than geographic origin $7,19-21$, whereas other studies suggest that geographic origin is more significant ${ }^{13,22}$. Furthermore, previous research has yielded differing opinions on the genetic diversity of $B$. napus, including an ongoing debate over which type of $B$. napus (winter or spring, rapeseed, or rutabaga) exhibits higher nucleotide diversity $7,20,23,24$. However, one consistent observation is that the $\mathrm{A}$ and $\mathrm{C}$ sub-genomes of $B$. napus have different degrees of genetic diversity ${ }^{7,20}$.

Understanding the origin and diversification of B. napus is important for germplasm preservation and for breeders to develop heterotic groups ${ }^{25}$. Therefore, we perform a comprehensive study of
183 accessions of $B$. napus and 174 accessions of potential progenitors. These include representatives of the major morphotypes across a global geographic distribution to determine the origin of $B$. napus by analyzing genomic SNPs and performing de novo assemblies of chloroplast and mitochondrial genes. We investigate $B$. napus selective sweep regions, and discuss differentially expressed genes (DEGs) highly related to the diversification process of $B$. napus. We further identify candidate genes under selection and unique DEGs for functional analysis associated with root tuber formation, leaf shape, and vernalization.

\section{Results}

RNA-seq and SNP identification. Our dataset includes $183 \mathrm{~B}$. napus accessions, $112 \mathrm{~B}$. rapa accessions, $42 \mathrm{~B}$. oleracea accessions, $20 \mathrm{~B}$. oleracea wild relatives (B. hilarionis, $B$. villosa, $B$. montana, B. macrocarpa, B. rupestris, B. incana, B. insularis), and five other Brassicaceae species as outgroups (Supplementary Data 1). Collectively these represent the main phenotypic diversity in each species. For B. napus, this panel represents all the major production countries and phenotypes: spring rapeseed, winter rapeseed, semi-winter rapeseed, rutabaga, and Siberian kale. Quality-filtered reads were mapped to the B. napus Darmor$b z h$ reference genome (version 4.1$)^{1}$ with an average overall mapping rate of $77.28 \%$ in B. napus, $45.73 \%$ in B. rapa, $63.91 \%$ in $B$. oleracea and its wild relatives, and $22.80 \%$ in outgroups. This indicates that the $\mathrm{C}$ genome is more conserved than the $\mathrm{A}$ genome in $B$. napus, consistent with previous studies ${ }^{7,20}$.

We obtained 372,546 high-quality SNPs across all B. napus accessions for diversity and selection analyses. 95.03\% (354,033) of these SNPs were located in genes, which matches our expectation for RNA-Seq data. The A sub-genome contained $219,164(58.83 \%)$ SNPs and the C sub-genome comprised the remaining $153,382(41.17 \%)$ SNPs. The C sub-genome is larger than the A sub-genome; however, more SNPs are found in the A sub-genome due to its higher nuclear diversity. The SNP density varied from 0.286 per $\mathrm{kb}$ on chromosome $\mathrm{C} 02$ to 1.080 per $\mathrm{kb}$ on chromosome A03. The most and fewest SNPs were found on A09 $(29,627)$ and C02 $(13,240)$, respectively (Supplementary Table 1). After further filtering, 36,829 synonymous and non-coding SNPs for $B$. napus were identified and used for genetic structure analysis. Then we added outgroups to all $B$. napus accessions and filtered the SNPs with the same pipeline to do the phylogenetic analysis. Similarly, 24,193 and 23,387 synonymous and noncoding SNPs were used to construct the A sub-genome and C sub-genome phylogenies, respectively.

We also used the rapeseed pan-transcriptome ${ }^{26}$ as a reference to recover 47,553 synonymous and non-coding SNPs across all $B$. napus accessions. As with the Darmor-bzh genome, the A genome (25,569 SNPs) showed more SNPs than the C genome (21,984 SNPs). To assess the role of the reference in determining the genetic clusters, these SNPs were also used to test for B. napus genetic structure and phylogenetic relationships.

Genetic structure and phylogenetic relationship. FastSTRUCTURE $^{27}$ was used to investigate the genetic structure of $B$. napus for clusters $(K)$ from 1 to 10 based on the two reference sequences. When applied to the SNPs based on Darmor-bzh genome $(36,829$ SNPs), we found that the marginal likelihood plateaued at $K=5$, and the cluster best fit for this data was identified as $K=7$ (Supplementary Fig. 1). At the plateau of marginal likelihood, we identified five genetic clusters, including Winter rapeseed in Europe and America (WEAm), Rutabaga (R), Spring rapeseed (S), Siberian kale (SK), and Winter rapeseed in East Asia (WeA, or semi-winter rapeseed) (Fig. 1a, Supplementary Fig. 2, Supplementary Data 2). After that, the marginal likelihood value slightly 
a

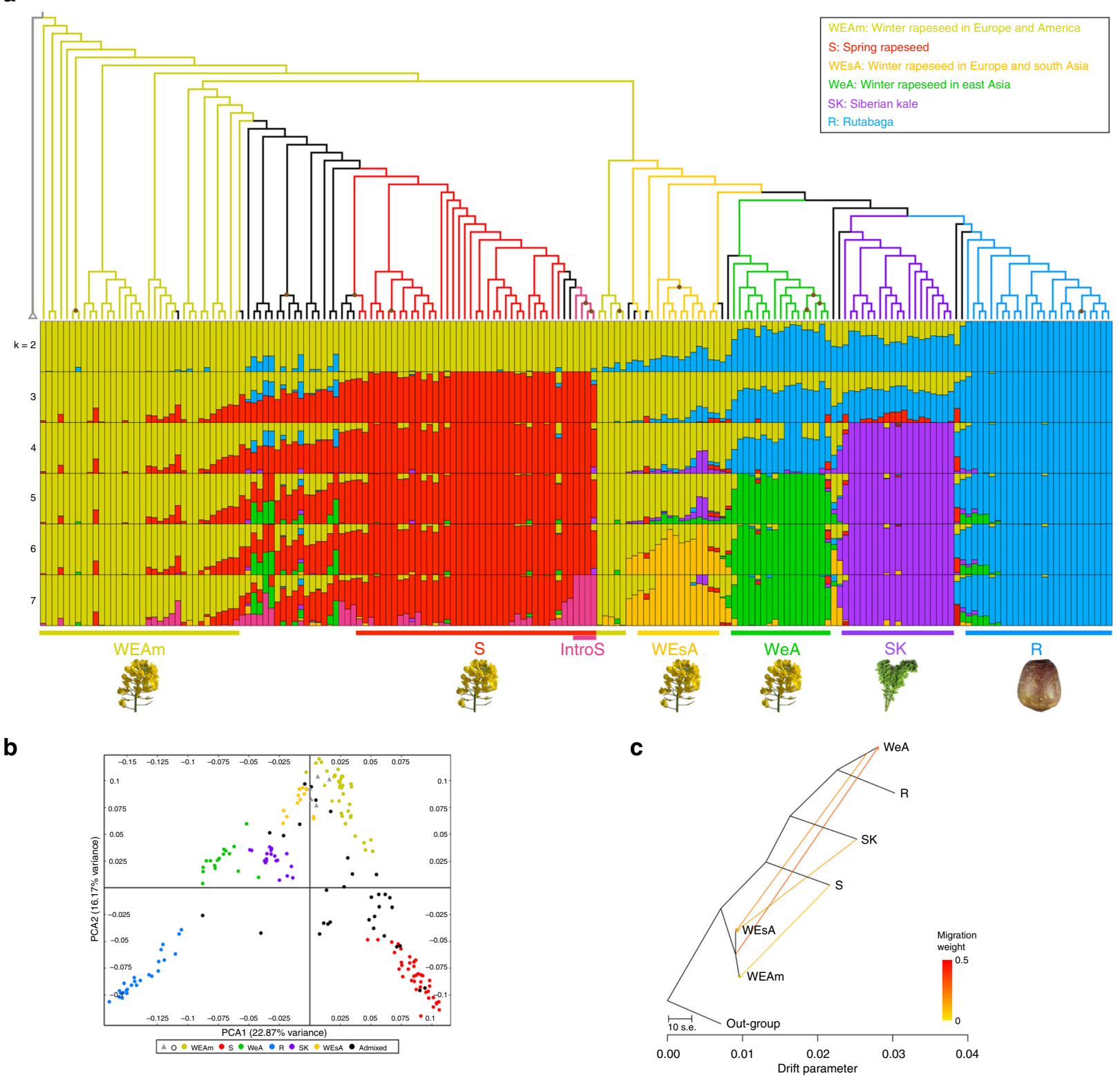

Fig. 1 Population genetics analyses of 183 B. napus accessions. a Genetic structure and maximum-likelihood phylogeny of $B$. napus. Five other species from Brassicaceae used to root the phylogenetic tree are shown as a single branch. The fastSTRUCTURE ancestry kinship (K) is shown from 2 to 7 . Colors indicate different genetic clusters. Yellow: WEAm; Red: S; Orange: WEsA; Green: WeA; Purple: SK; Blue: R; Pink: IntroS (Introgressed spring rapeseed); Black: genetically diverse accessions; Dark grey: outgroup. Brown dot: bootstrap support values $<70 \%$. b Principal component analysis of the 183 B. napus and five outgroup accessions. The B. napus color chart is the same as in $\mathbf{a}$. Grey triangles were added to represent outgroups. $\mathbf{c}$ Treemix analysis of the six $B$. napus genetic clusters. Arrows represent the direction of migrations. Source data of Fig. 1a are provided as a Source Data file

increased at $K=6$ and 7 , and reached the peak at $K=7$. When $K$ $=6$, Winter type rapeseed in Europe and South Asia (WEsA) were observed to cluster together. We found that at $K=7$ that four spring type accessions (marked as IntroS) appeared to share genetic structure with other spring rapeseed; however, they did not differ from the other spring type accessions in phenotype or geographic location and were therefore kept in the S cluster. In addition to these six main clusters, some genetically diverse accessions (the main genetic component was less than 0.6 in fastSTRUCTURE) were also identified in our analyses; some of these accessions were recently admixed or resynthesized during the breeding process.

The genetic structure based on using the rapeseed pantranscriptome as a reference for mapping (47,553 SNPs) also showed that the marginal likelihood plateaued at $K=5$, and identified the same five genetic clusters as described above (Supplementary Fig. 3, Supplementary Fig. 4). Interestingly, the IntroS, observed at $K=7$ with the Darmor-bzh genome (Fig. 1a), is observed at $K=6$ in the analysis based on the rapeseed pantranscriptome (Supplementary Fig. 4). This IntroS may be the result of introgression as indicated by the chloroplast data described below. Thus, using either the Darmor-bzh or pantranscriptome as a reference, we recover the same six genetic clusters with increasing $K$ (Fig. 1a, Supplementary Fig. 4). Given this agreement in the major clustering from both analyses, we suggest the recognition of six genetic clusters in $B$. napus as: WEAm, R, S, SK, WeA, and WEsA. 
Using five closely related species (Brassica nigra, Crambe hispanica, Sinapis alba, Eruca sativa, and Sisymbrium irio) as outgroups, a maximum-likelihood phylogeny was constructed to resolve the relationships among the different $B$. napus subspecies (Fig. 1a). The phylogeny is highly congruent with our genetic structure results of six clusters, with two paraphyletic groups (WEAm and WEsA) and four monophyletic groups (R, S, SK, and WeA). Using the Darmor-bzh genome, we recover the WEAm cluster as sister to all other B. napus subspecies. The other five genetic clusters (S, WEsA, WeA, SK, and R), identified in the fastSTRUCTURE analysis, form two clades, each containing some WEAm accessions and other genetically diverse accessions. One of these grades contains all the $S$ accessions and the other is composed of R, SK, WeA, and WEsA genetic clusters. A second phylogeny using the same outgroups, but with the resulting SNPs from mapping to the rapeseed pan-transcriptome ${ }^{26}$ was constructed. It showed the same two paraphyletic groups (WEAm and WEsA) and the same four monophyletic groups (R, S, SK, and $\mathrm{WeA}$ ), as well as all $\mathrm{S}$ accessions in a clade sister to all other winter type B. napus (WEAm, R, SK, WeA, and WEsA; Supplementary Fig. 5). However, we also noticed some inconsistent patterns between these two phylogenies (Fig. 1a, Supplementary Fig. 5), including the relationships among R, SK, and $\mathrm{WeA}$, and the placement of WEAm accessions.

In summary, using both references, we show the same six genetic clusters: two paraphyletic groups (WEAm and WEsA) and four monophyletic groups (R, S, SK, and WeA). Given the similarity of these results, we feel confident in using six groups for the remaining analyses below.

The genetic structure and phylogenetic relationships were also congruent with results from the principal component analysis (PCA) (Fig. 1b). In the PCA plot, all accessions assigned to clusters for B. napus were found to cluster into the six previously identified groups. These lineages' migration histories was examined among the six genetic clusters using Treemix ${ }^{28}$, which implements composite likelihood to identify the most likely population splits. Individuals that phylogenetically clustered and had a consistent phenotype with these six genetic clusters were used in these analyses. Without migration $(\mathrm{m}=0)$, the Treemix result explained $85.51 \%$ of the variance in SNP data. When we added migration events for four migration edges $(\mathrm{m}=4)$ we were able to account for $99.74 \%$ of the variance (Fig. 1c). This graph model inferred four gene flows: from $S$ to WEAm, from SK to WEsA, from WeA to WEsA, and from WEAm to WeA (Fig. 1c). This observation may explain the genetically diverse accessions in the genetic structure results.

Overall, the genetic structure and PCA of B. napus was found to correspond more to morphotype and growth habits than geographic origin, which is similar to other studies $7,19,20$. Consistent with insights from previous studies of $B$. napus origin $^{1,3,18}$, our phylogeny based on Darmor-bzh genome also indicates that the WEAm genetic cluster was most likely the first formed morphological subgroup. The order of the genetic clusters fall out in the fastSTRUCTURE analysis is consistent with written records: the first references of $B$. napus are winter rapeseed (1578), rutabaga (1620), and spring rapeseed $(\sim 1700)^{8,11}$. However, while our inferred phylogeny and those from previous studies generally agree on the genetic clusters, they are incongruent with each other with respect to the relationships among these clusters $4,12,13,16$. This divergence may be caused by the different germplasm sets used, the variety of methods employed, or different reference sequences used in these studies. The inconsistent relationship of rutabaga (R), Siberian kale (SK), and semi-winter rapeseed (WeA) indicates that a $B$. napus pan-genome that can represent all the morphotypes is greatly needed. With these genomes, we can access homoeologous genome exchanges among different subspecies easier. Admixture was widespread among different $B$. napus clusters, especially in rapeseed subspecies, which may be due to efforts by breeders or the result of different genetic clusters being grown in close geographic proximity. Breeder-associated admixture between different genetic clusters has often been used to create heterosis and disease resistance; for example, crossing spring or European winter lines with Chinese semi-winter lines ${ }^{29,30}$.

Relationships among Brassica napus and its progenitors. We generated two maximum-likelihood (ML) phylogenies based on the $\mathrm{A}$ and $\mathrm{C}$ sub-genome separately. Based on the A sub-genome phylogeny, we studied the relationship between $B$. napus and 112 accessions of $B$. rapa. The phylogeny again showed six genetic clusters among $B$. napus samples and together are sister to a monophyletic B. rapa. A newly resynthesized $B$. napus accession New Hakuran was found to cluster with $B$. rapa, a pattern, which is known to occur with newly formed accessions ${ }^{13}$ (Fig. 2a, Supplementary Fig. 6). Based on the $C$ sub-genome, we investigated the relationship between $B$. napus and 62 accessions of $B$. oleracea as well as the wild relative species. Although the wild $C$ species and B. oleracea cultivars were clearly separated into two branches, they were recovered as a monophyletic group sister to a $B$. napus clade excluding the newly resynthesized New Hakuran and a Siberian kale accession (Fig. 2b, Supplementary Fig. 7). When compared with the $\mathrm{A}$ and $\mathrm{C}$ sub-genome phylogenies constructed based on the rapeseed pan-transcriptome, incongruences were found (Supplementary Fig. 8). Since $88.22 \%$ $(102,422$ out of 116,098$)$ CDS models of the rapeseed pantranscriptome are from $B$. rapa and $B$. oleracea genomes ${ }^{26}$, it indicates that the components of the reference sequences have non-negligible impact on the topology of phylogenies. However, although the relationships among these clusters differed, all analyses again consistently recovered six genetic clusters in both the $\mathrm{A}$ and $\mathrm{C}$ sub-genome phylogenies with the both reference sequences.

We also explored the relationships between $B$. napus and its progenitors based on each chromosome. These 19 phylogenies mainly showed the same species level relationships as the subgenome except three $\mathrm{C}$ sub-genome chromosomes C01, C02, C05 (Supplementary Fig. 9). This may be the result of recent subgenome rearrangement, as $\mathrm{C} 01$ and $\mathrm{C} 02$ have the most frequent homoeologous exchanges ${ }^{31}$. Another possibility for this phenomenon could be interspecies introgression, as seen from Raphanus raphanistrum to $B$. napus ${ }^{32}$.

Subsequently, the chloroplast and mitochondrial genes of $B$. napus and progenitors were de novo assembled using data generated by genome survey sequencing (GSS). We constructed a ML phylogeny with both chloroplast and mitochondrial data. In the chloroplast phylogeny, two wild $C$ species, $B$. villosa and $B$. rupestris, were found to be sister to all other species. Additionally, the $B$. napus clade was separate from $B$. oleracea, wild $C$, and $B$. rapa (Fig. 2c, Supplementary Fig. 10). Unlike the nuclear genome, we were generally unable to distinguish subspecies based on the chloroplast phylogeny: the only subspecies that was monophyletic was rutabaga (R). In addition, some $B$. napus accessions clustered in the $B$. rapa clade or $B$. oleracea clade and vice versa. It is interesting to note that IntroS was classified in the B. rapa clade (bootstrap support $>70 \%$; Fig. 2c, Supplementary Fig. 10). This association suggests a recent introgression between $B$. rapa and $B$. napus, since spring rapeseed is a recently formed subgroup. This introgression event makes this small spring rapeseed group different from other spring rapeseed in both its nuclear and organellar genome. The mitochondrial phylogeny, similar to the chloroplast phylogeny, showed the wild $\mathrm{C}$ species as a single 
a
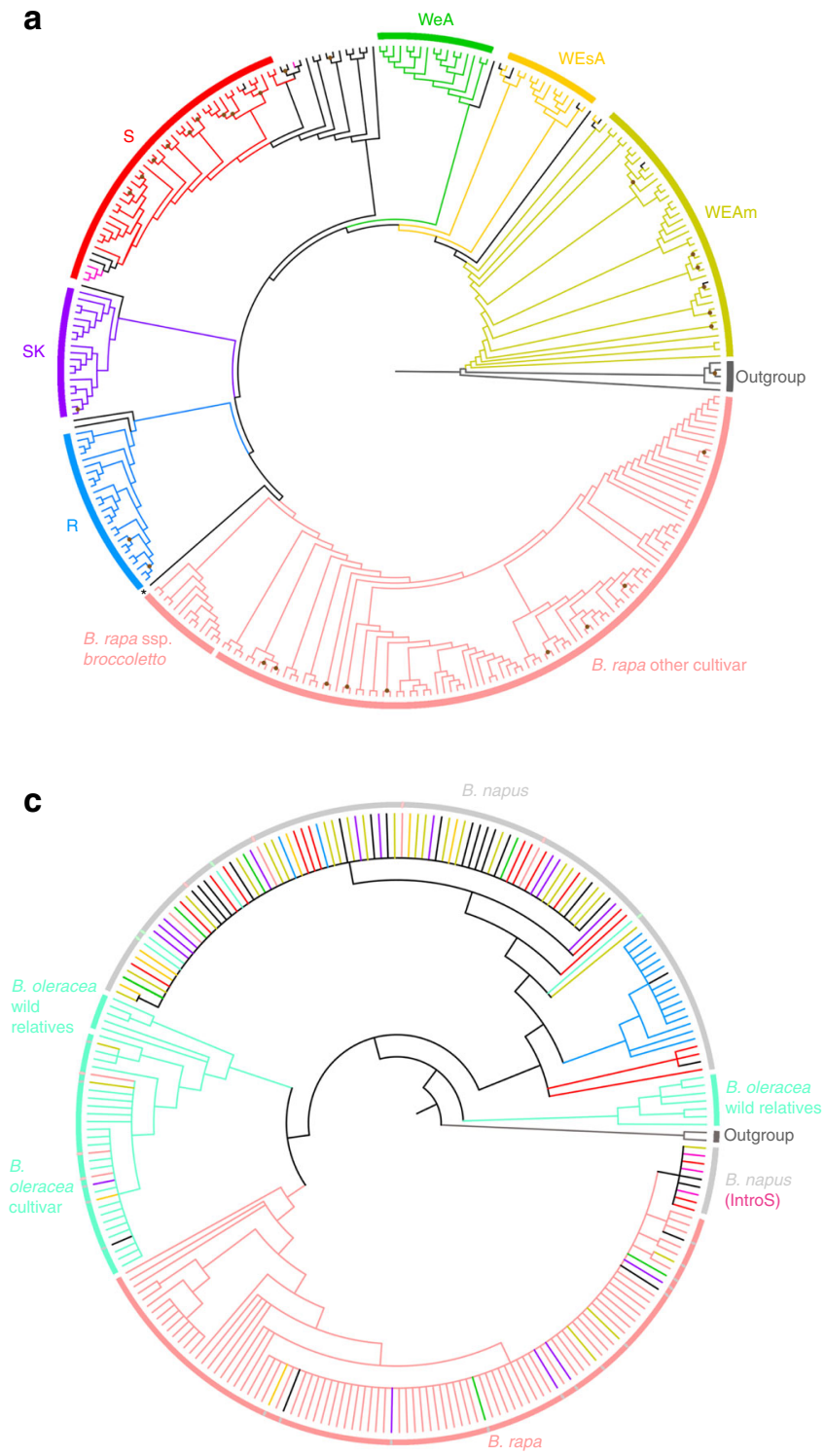

b

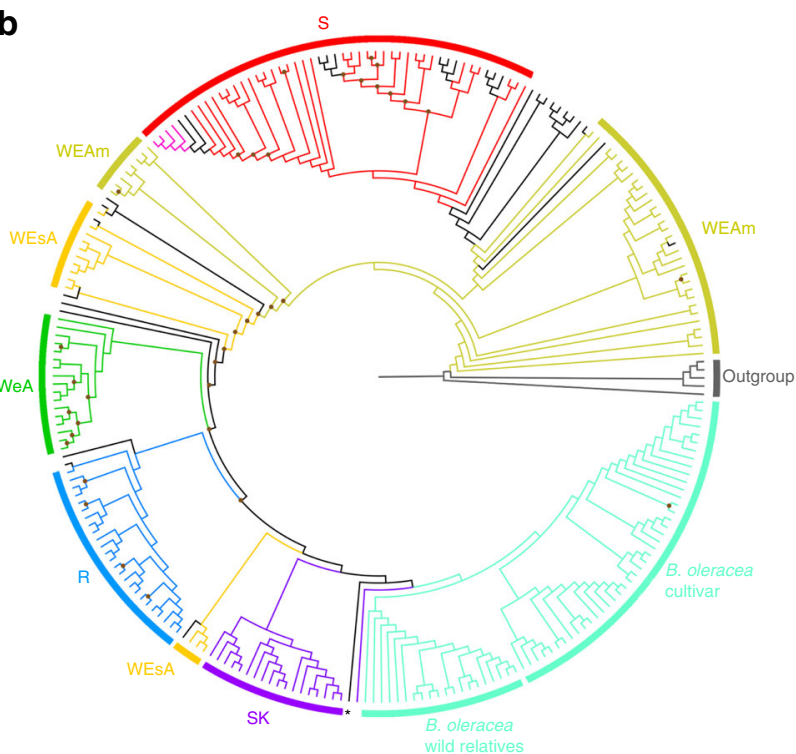

d

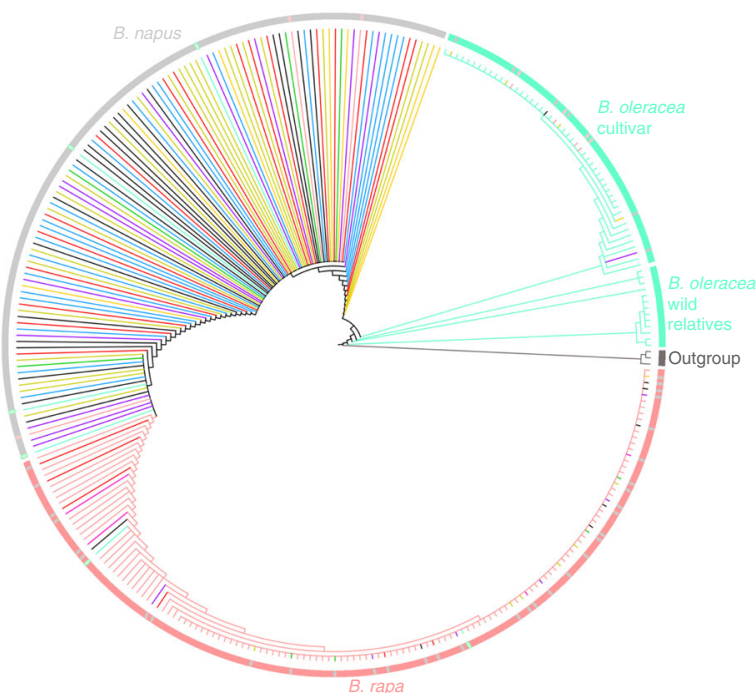

Fig. 2 Phylogenetic analyses of $B$. napus and its progenitors. Maximum-likelihood phylogenetic tree constructed among: a $B$. napus and $B$. rapa based on the A sub-genome (24,193 SNPs); b B. napus, $B$. oleracea, and wild $C$ species based on the $C$ sub-genome (23,387 SNPs); c Chloroplast phylogeny of $B$. napus and all its progenitor species (B. rapa, B. oleracea, and wild $C$ species) using 62 chloroplast genes; d Mitochondria phylogeny of $B$. napus and all its progenitor species using 42 mitochondrial genes. *: resynthesized B. napus accession New Hakuran; WEAm: Winter rapeseed in Europe and America; $S$ spring rapeseed, WEsA winter rapeseed in Europe and South Asia, WeA winter rapeseed in East Asia, SK Siberian kale, R rutabaga, IntroS Introgressed spring rapeseed. Brown dot: bootstrap support values $<70 \%$; c, d were made with a bootstrap cut off value of $70 \%$

clade. The remaining samples clearly divided into three clades: $B$. oleracea, B. napus, and B. rapa (Fig. 2d, Supplementary Fig. 11). Some mixed clusters among the three species were also observed.

The proposed multiple origins of B. napus is not confirmed based on the nuclear phylogenies, which is consistent with previous studies using nuclear markers ${ }^{13,15}$. Similar to Song et al. (1992) ${ }^{13}$, we found only newly resynthesized B. napus clustered directly with its progenitors. Our results suggest that perhaps we have not sampled the extant populations of $B$. rapa and $B$. oleracea that are most similar to the ancient progenitors of $B$. napus, or that the progenitor populations may be extinct ${ }^{33}$. Alternatively, it is also possible that after several thousand years of evolution, the B. napus nuclear genome has diverged beyond easy recognition from its progenitors' nuclear genomes. The topological difference of these six genetic clusters in $\mathrm{A}$ and $\mathrm{C}$ sub-genomes also indicates that there was asymmetric sub-genome evolution during their diversification. Compared to the nuclear phylogenies, the organellar phylogenies of $B$. napus are too conserved to distinguish these six genetic clusters. Interestingly, our chloroplast result matches the nuclear genome-based Treemix ${ }^{28}$ result, which showed no significant admixture between rutabaga (R) and other genetic clusters. Our chloroplast results differ from previous studies that found a multi-origin of $B$. napus using haplotypes of the chloroplast genome $\mathrm{e}^{13,15}$. It is common for breeders to cross $B$. rapa with $B$. napus to make $B$. napus more fit in local environments or have better agronomic traits 34,35 , which may contribute to the introgression identified in the chloroplast phylogeny. Apart from known crosses, it is difficult to differentiate mixed clusters found among B. napus and its progenitors are due to natural hybridizations or breeder mediated introgression. 
a

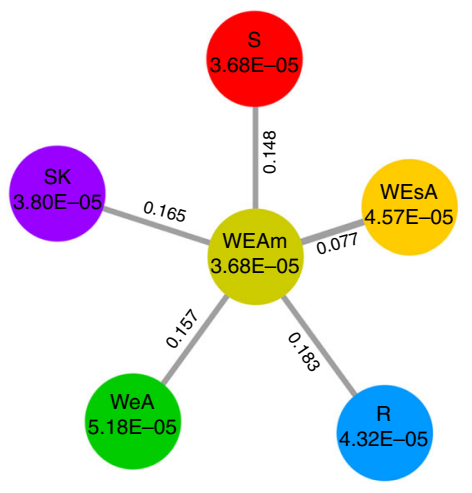

b

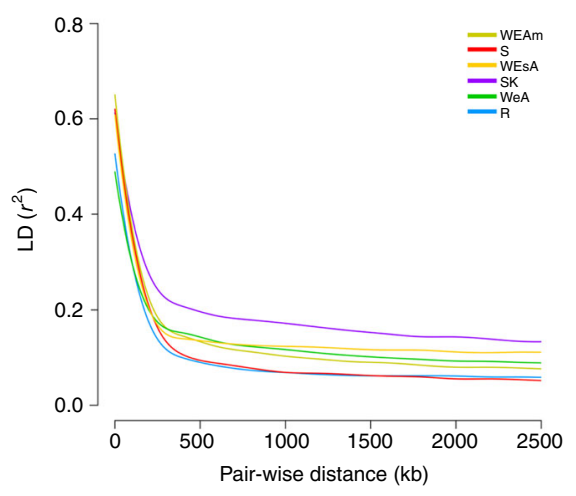

c

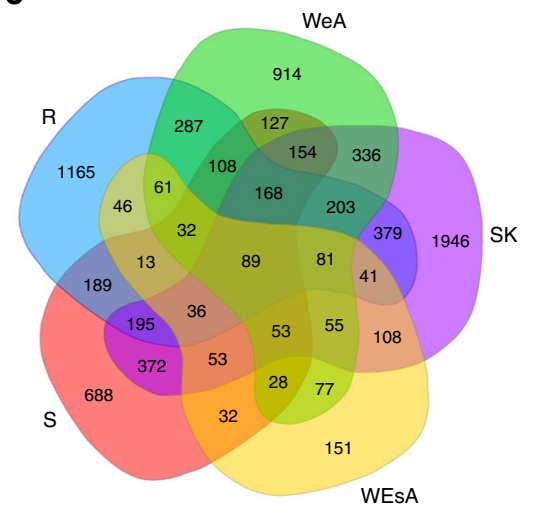

d

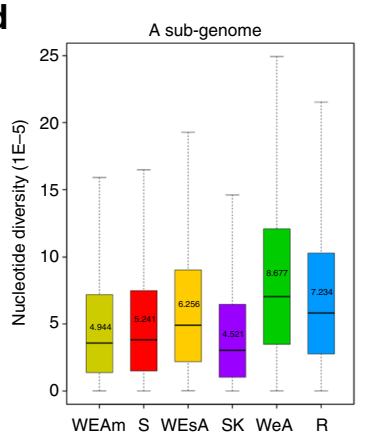

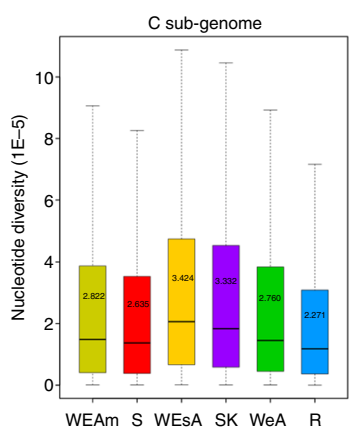

e

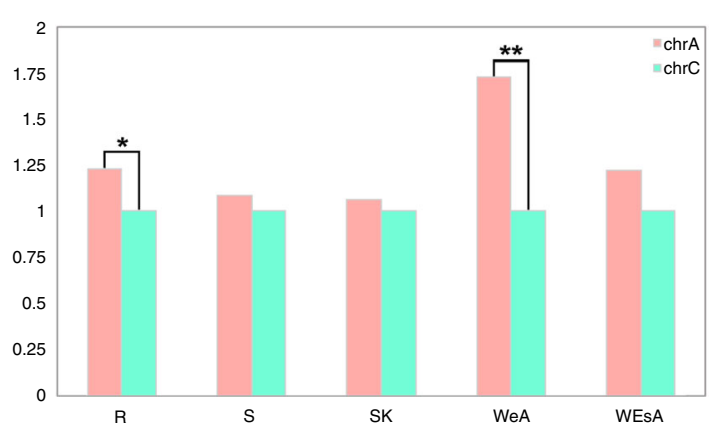

Fig. 3 Comparison of different B. napus genetic clusters. a Nucleotide diversity and $\mathrm{F}_{\mathrm{ST}}$ across six genetic clusters. The value in each circle shows nucleotide diversity for this cluster; the value on each line represents the $F_{S T}$ between WEAm and another cluster. $\mathbf{b}$ Decay of linkage disequilibrium (LD) in each genetic cluster measured by $r^{2}$. c Venn diagram of differentially expressed genes (DEGs) between WEAm and other genetic clusters. $\mathbf{d}$ Nucleotide diversity in each genetic cluster based on separated $A$ and $C$ sub-genomes. The box represents the interquartile range. Band inside each box represents median. Number inside each box represents the mean value. The whiskers represent the minimum and maximum values. e The ratio of (DEGs in A sub-genome)/ (DEGs in C sub-genome). ${ }^{\star} p$-value $<0.05$ ( $\chi^{2}$ test); ${ }^{\star \star} p$-value $<0.01$ ( $\chi^{2}$ test). 372,546 SNPs were used for all the analysis in $\mathbf{a}, \mathbf{b}$, d. Source data of Fig. $3 e$ are provided as a Source Data file

Nucleotide and gene expression changes via diversification. We examined population divergence among the six different genetic clusters by calculating their fixation statistics $\left(\mathrm{F}_{\mathrm{ST}}\right)$. The results indicate Rutabaga $(\mathrm{R})$ had the largest population divergence from WEAm (0.183), while WEsA was found to have minimum divergence from WEAm (0.077) (Fig. 3a, Supplementary Table 2, Supplementary Fig. 12). Based on nucleotide diversity $(\pi)$ for each genetic cluster, we found WEAm and spring rapeseed $(S)$ had the lowest and equal nucleotide diversity $\left(\pi=3.68 \times 10^{-5}\right)$, closely followed by Siberian Kale (SK) $\left(\pi=3.80 \times 10^{-5}\right)$, while WeA showed the highest nucleotide diversity $\left(\pi=5.18 \times 10^{-5}\right)$ (Fig. 3a). Linkage disequilibrium (LD) analyses revealed that SK had the largest LD value, followed by WEAm, WEsA, and WeA (the three winter rapeseed clusters); $\mathrm{R}$ and $\mathrm{S}$ had the lowest LD value (Fig. 3b).

The gene expression analysis in leaf tissue between WEAm and five other genetic clusters showed that 8,187 genes were differentially expressed genes (DEGs). SK had the most DEGs $(4,269)$ with R $(3,093)$ also showing a high number of DEGs (Supplementary Data 3, Supplementary Table 3). WeA and S had similar DEGs with 2,773 and 2,337 genes separately. Meanwhile, WEsA, whose phenotype was more similar with WEAm, only had 956 DEGs. Since R and SK clusters had observably different morphotypes with WEAm, $\mathrm{S}$ and WeA had distinguishable growth habits with WEAm, these results were reasonable. Interestingly, there were significantly more downregulated DEGs than up-regulated DEGs $(t$-test, $p<0.05)$ (Supplementary Table 3 ). We found 4,864 DEGs were unique to different genetic clusters. Following a similar pattern to the total DEGs in each cluster, SK $(1,946)$ and $\mathrm{R}(1,165)$ had more unique DEGs than WeA (914) and S (688), and WEsA (151) had fewer unique DEGs than the others (Fig. 3c). When using the rapeseed pan-transcriptome, the same patterns as described above were identified, suggesting that while differences in the reference sequence have profound effects on phylogeny, it does not at population level analyses. (Supplementary Fig. 13). The number of DEGs varied among chromosomes as well. Generally, A03, A09, C03 had more DEGs than the others and A04, A08 had fewer DEGs (Supplementary Fig. 14).

Nucleotide diversity and DEGs were analyzed based on A and C sub-genomes as well. Generally, the A sub-genome had higher genetic diversity than the $\mathrm{C}$ sub-genome (Fig. $3 \mathrm{~d}$ ), again agreeing with previous studies ${ }^{7,20}$. Based on the A sub-genome, WeA had the most genetic diversity while SK had the least (Supplementary Table 4). However, based on the C sub-genome, WEsA and R showed the highest and lowest nucleotide diversity, respectively. Besides, the A sub-genome obtained more DEGs than the C subgenome, especially in WeA and $\mathrm{R}\left(\chi^{2}\right.$ test, $p<0.01$ and $p<0.05$, respectively) (Fig. 3e). The genetic diversity and DEGs difference in the $\mathrm{A}$ and $\mathrm{C}$ sub-genome among each genetic cluster also indicated asymmetric sub-genome evolution during their diversification. This phenomenon is also reported in paleopolyploid $B$. oleracea and allotetraploid $\operatorname{cotton}^{36,37}$. The unique DEGs obtained in each genetic cluster may play significant roles in their differences in growth habit or morphotype.

Previous studies have found inconsistent rankings of genetic diversity among different subgroups ${ }^{7,20,23,24}$. Bus et al. (2011) found that the recently released winter rapeseed inbred lines have 

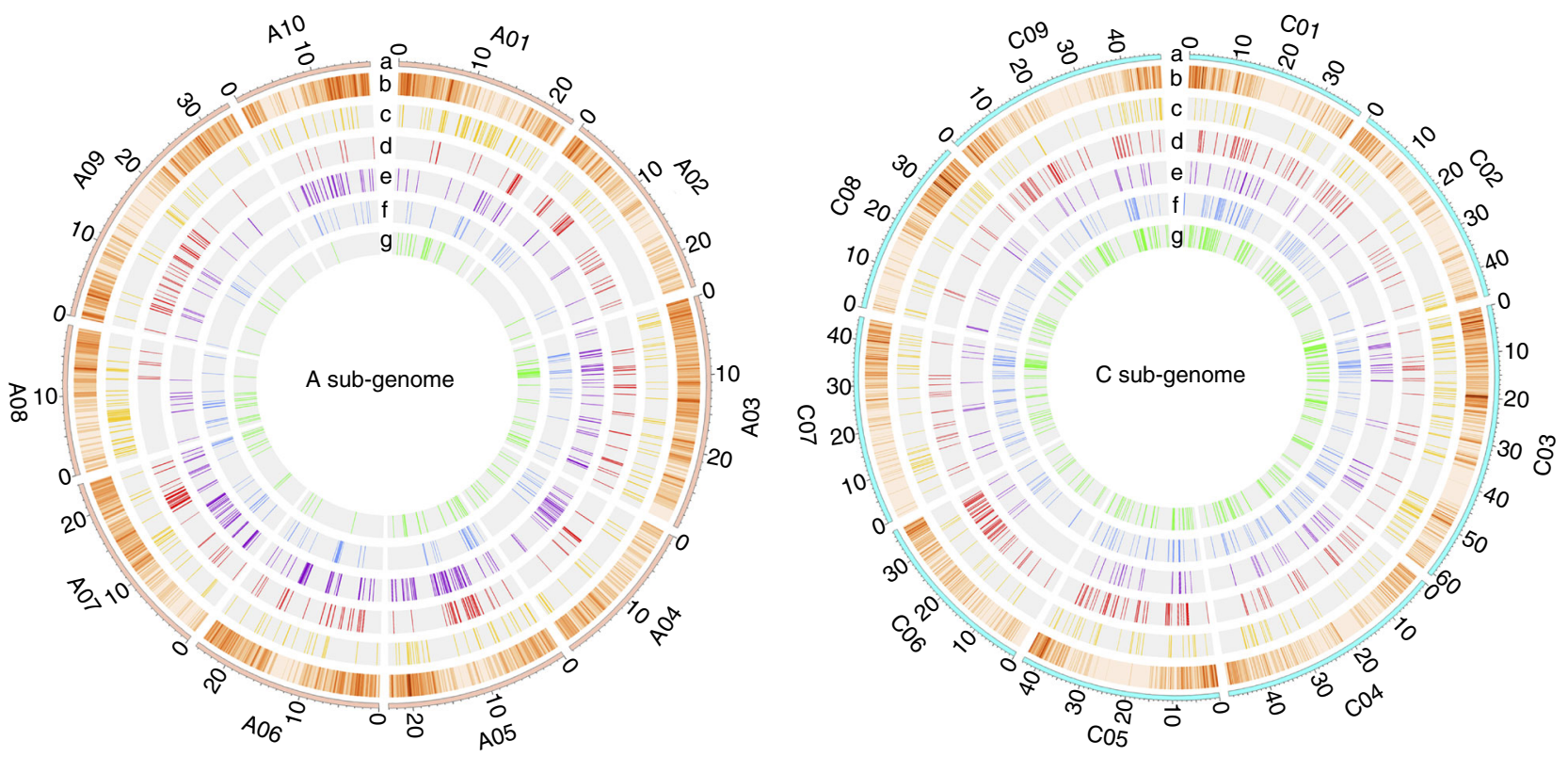

Fig. 4 Selective sweep regions among different genetic clusters. Left panel is $A$ sub-genome and right panel is $C$ sub-genome, each with rings labeled a-g. a $A$ and $C$ chromosomes of $B$. napus. b SNP density within a $200 \mathrm{~kb}$ window across each chromosome. c-g selective sweep regions. Colors indicate different genetic clusters. Red: S; Orange: WEsA; Green: WeA; Purple: SK; Blue: R

lower genetic diversity than lines of winter rapeseed that have been inbred and released several decades ago ${ }^{24}$. In our study, both Winter rapeseed in Europe and America (WEAm) and spring rapeseed (S) have the lowest genetic diversity. This low diversity may have been caused by intensive breeding for better seed quality, like double-low rapeseed, followed by huge population expansion in Europe and Canada ${ }^{38,39}$. The selective pressures experienced by spring rapeseed (S) may also explain the faster $\mathrm{LD}$ decay in S compared to winter rapeseed, a hypothesis consistent with Delourme et al. $(2013)^{23}$. The other genetic clusters with higher genetic diversity may be due to the recent introgression by breeders. For example, when breeders introduced $B$. napus to East Asia, they performed crosses of $B$. rapa to $B$. napus to generate $B$. napus cultivars that were better adapted to the local environment ${ }^{40}$. These breeding practices most likely contributed to WeA having the most genetic diversity in the A sub-genome.

Identification of selective sweep regions. The diversification of $B$. napus resulted in changes in morphotype and growth habit, including variation in root expansion, leaf shape, and vernalization requirements. A total of 570, 474, 452, 420, and 412 selective sweep regions were both identified by XP-CLR and $\pi$ ratio $\left(\pi_{\text {WEAm }} / \pi_{\text {other_cluster }}\right)$ methods in WEsA, S, WeA, R, and SK, which contained 3,391,2,972,2,619,2,414, and 2,447 genes, respectively (Fig. 4, Supplementary Data 4). Although WEsA had the most selective sweep regions, the top 5\% XP-CLR score (7) for WEsA is much lower than others (SK: 15, R: 14, S: 12, WeA:11) (Supplementary Fig. 15).

We were able to identify genes, which were important for the different morphotypes or growth habits in our selective sweeps. For example, we identified BnaA06g18280D and BnaC03g38180D whose homologs in its potential progenitor (Bra038270, Bo3g065780) functioned in tuber-forming in rutabaga $(\mathrm{R})^{41}$. Additionally, 19 other candidate selected genes were identified that may function in forming tuberous roots in rutabaga, including three genes regulating auxin signaling, one gene functioning in auxin transport, and one gene contributing to auxin synthesis in roots (Supplementary Table 5, Supplementary
Fig. 16). In leafy type Siberian kale (SK) we found BnaA05g16600D, which is homologous to candidate genes identified in B. rapa (Bra033869) and Arabidopsis thaliana (AT1G31880) that contribute to the curled leaf phenotype ${ }^{41}$. In addition, 10 selected genes were identified in SK, which are highly related to leaf shape and two genes functioning in cellular response to auxin stimulus (Supplementary Table 6, Supplementary Fig. 17). Additionally, five genes that function in flowering time and vernalization, BnPHYA, BnEFS, BnSUF4, BnSPL3, and BnaA07g22720D $7,42,43$, were found in selective sweep regions of spring rapeseed (S). Another 18 candidate genes that are believed to possibly regulate flowering time or vernalization in $S$ were identified (Supplementary Table 7, Supplementary Fig. 18). Interestingly, among all these candidate genes we only identified one paralogous gene pair (BnaA06g18130D and BnaC03g55810D) in rutabaga $(\mathrm{R})$, which are both under selective sweeps. Similar to the DEGs, many selective sweep regions were specific to a genetic cluster. Only 22 genes among the six selective sweep regions were found to be common among all genetic clusters (Supplementary Fig. 16, Supplementary Table 8). Even though most of these genes (21 of 22) are homologous with genes belonging to $B$. rapa and $B$. oleracea, more than half ( 12 of 22 ) of these genes were unable to be annotated with $A$. thaliana genes. This indicates that many of these important crop diversification related genes are Brassica specific.

More selective sweep regions were found in the $\mathrm{C}$ sub-genome than A sub-genome among all genetic clusters except SK (Supplementary Fig. 17). This implies that $\mathrm{C}$ sub-genome has undergone stronger selection than the A sub-genome, which is consistent with a previous study in rapeseed ${ }^{40}$. The numbers of selective sweeps also varied between different chromosomes across the genetic clusters. These results imply that A09, C05, and C06 may have had more selective pressure during the change of growth habit in B. napus (Supplementary Fig. 18).

Of the top 5\% XP-CLR score, WEsA was found to have the lowest score compared to the other genetic clusters indicating most of the selective sweep regions in WEsA may play a role in crop improvement. In contrast, the other genetic clusters, with 


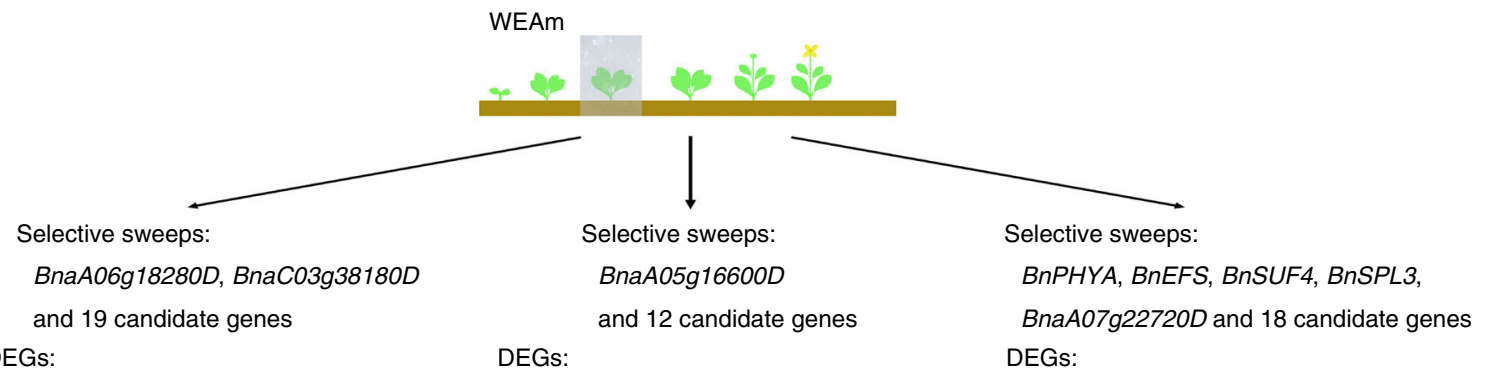

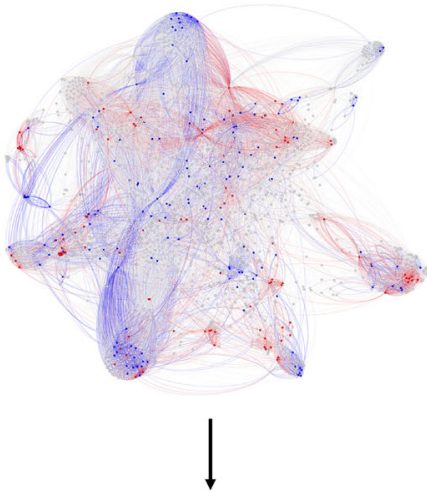

$\mathrm{R}$

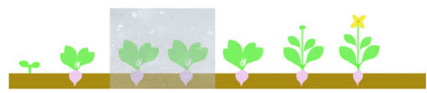

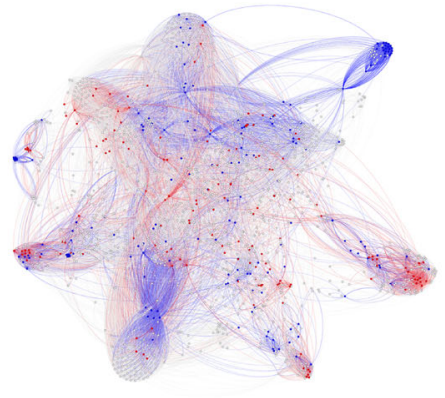

$\downarrow$

SK

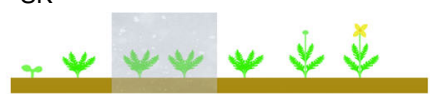

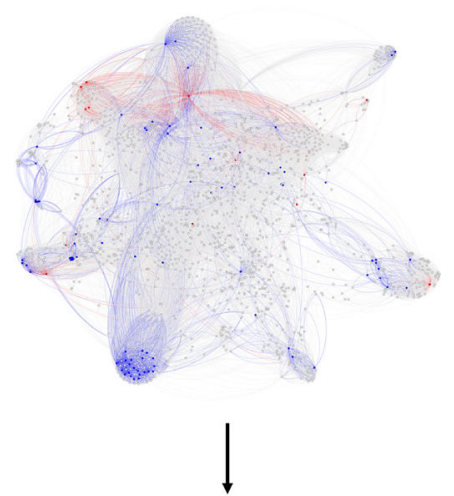

S

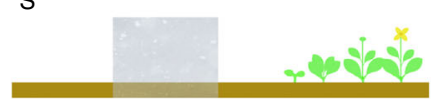

Fig. 5 Different diversification processes of B. napus. Plant cartoons were modified from Schiessl et al. (2017) ${ }^{43}$ and show the growth cycle of different $B$. napus, the grey rectangle indicates winter (vernalization). The networks represent biochemical reactions that are catalyzed by enzymes (three middle panels); the colors of the nodes (reactions) in the metabolic networks represent the difference in expression of the B. napus genes that are orthologs to the A. thaliana genes encoding the enzymes for these reactions. Red: overexpressed comparing to WEAm; Blue: underexpressed comparing to WEAm; Grey: no unique DEGs detected. WEAm: Winter rapeseed in Europe and America, S: spring rapeseed, SK: Siberian kale, R: rutabaga

top 5\% XP-CLR scores at least 11, contained more selective sweep regions contributing to the diversification of economically important organs such as the expanded root (rutabaga) or edible leaf (Siberian kale). Similar phenomena were also found in soybean $^{44}$. Brassica rapa, B. oleracea, and B. napus all have root type (like turnip, kohlrabi, and rutabaga) and leafy type (like Chinese cabbage, kale, and Siberian kale) vegetables ${ }^{11,18,41}$. As in a previous study of $B$. rapa and $B$. olerace ${ }^{41}$, we also found that the phytohormone, auxin, plays an important role in the new morphotype formation. Interestingly, the homologs of several of these genes in $B$. rapa and $B$. oleracea are also under positive selection during the domestication of tuber-forming and leaf curling ${ }^{41}$. These genetic clusters and genes can both be materials to study parallel evolution and be used in breeding processes ${ }^{45}$. Besides, these selective regions are good resources for specific agronomic trait introgression or admixture. CRISPR-Cas9 genome editing strategies may also be applied for these selected candidate genes for de novo diversifications of B. napus, similar to a study in tomato ${ }^{46}$. Our results showed $\mathrm{A}$ and $\mathrm{C}$ sub-genomes undergo different selective pressure, which indicates the asymmetric sub-genome evolution. Additionally, different chromosomes may have contributed unequally to different growth habits or morphotypes.

Comparative genomic analyses of different crops. We further compared the functions of unique DEGs among the three most diversified genetic clusters, spring rapeseed (S), rutabaga (R), and Siberian kale (SK), using Gene Ontology categories and information about metabolic reactions. In addition to cell metabolism or housekeeping genes, we identified DEGs in these three genetic clusters commonly related to three phytohormones (abscisic acid, cytokinin, and jasmonic acid), temperature (response to cold), and chloroplast-related genes (Fig. 5, Supplementary Data 5, Supplementary Data 6). Additionally, there are some clusterspecific categories or reactions significantly enriched in different genetic clusters. For example, genes associated with the function of positive regulation of flower development were upregulated in spring rapeseed (S), which correlates to its early flowering phenotype. In Siberian kale (SK), we found upregulated genes that function in response to different light (red, blue, and far red) and some downregulated genes related to glucosinolate biosynthesis. We speculate that this difference may be responsible for the dark green and tasty leaf of Siberian kale. In addition, some upregulated DEGs in rutabaga (R) were identified as functioning in glucosinolate biosynthetic and catabolic processes.

These preliminary results indicate that phytohormones play an important role in the diversification of $B$. napus which is consistent with Cheng et al. (2016) study in leaf-heading and tuber-forming in $B$. rapa and $B$. olerace ${ }^{41}$. Based on our phenotyping, spring rapeseed does not need vernalization, while rutabaga and Siberian kale need longer vernalization than winter rapeseed. Consequently, we found DEGs that function in response to cold in all three genetic clusters. Besides this global observation, the expression of some specific genes also changed due to different diversification processes, including the genes that regulate flower development in spring rapeseed and the genes that respond to different light in Siberian kale (SK). It is interesting that the glucosinolates and their products varied among different $B$. napus subspecies. This variation may be due to different artificial selection during their formation. For example, the expression of genes in the glucosinolate pathway was 
downregulated in Siberian kale, which may be due to consumers preferring leafy type vegetables without glucosinolates. Comparatively, rutabaga, a vegetable whose roots are consumed, may have high glucosinolate content in its leaf for better biotic resistance.

\section{Discussion}

In our comprehensive study of $B$. napus morphotypes, we identified six genetic clusters of $B$. napus consistently across genetic structure, PCA, and phylogenetic analyses, regardless of reference sequence used for mapping. Spring rapeseed was found to be independently derived from winter rapeseed in Europe from the other four winter types of $B$. napus. We were unable to confirm the multi-origin hypothesis of $B$. napus; however, our comparisons of nuclear and organellar data suggest past introgressions between $B$. napus and its possible progenitors, as well as admixture among different $B$. napus genetic clusters. The population genetic analyses showed that these six genetic clusters have undergone different selective pressures, which match the known breeding histories. Using both nucleotide diversity and gene expression level, we show that B. napus has an asymmetric subgenome evolution. We further identified candidate genes that went through selective sweeps underlying vernalization (spring rapeseed), leaf shape (Siberian kale), and root tuber-forming (rutabaga). The common and specific metabolic networks and GO functions we found among the main three morphotypes aids our understanding of $B$. napus diversification.

Our study can further facilitate B. napus breeding and germplasm preservation efforts. Candidate genes and pathways identified can be used for selecting agronomic traits. Genetic structure and phylogenetic results can assist breeders to find crossing groups to maximize heterosis. Finally, the high-quality SNPs can help breeders to conduct genome wide association studies and marker-assisted selection.

\section{Methods}

Sample collection and high-throughput sequencing. All the seeds used in this study were from U.S. Department of Agriculture (USDA), and the author's personal collection (Supplementary Data 1 ). Seeds were planted in a Conviron growth chamber $\left(16 \mathrm{~h}\right.$ of light at $23^{\circ} \mathrm{C}$ and $8 \mathrm{~h}$ of dark at $20^{\circ} \mathrm{C}$ ) with four replicates for each accession at the Bond Life Science Center at the University of Missouri (Columbia, MO, USA). The second youngest leaf of each plant was sampled and combined per accession for RNA and DNA isolation when the plants had five true leaves. After that, RNA was isolated using the PureLink ${ }^{\mathrm{TM}}$ RNA Mini Kit (Invitrogen, USA), double-stranded cDNA was synthesized though Maxima H Minus Double-Stranded cDNA Synthesis Kit (Thermo, Lithuania) and then purified by GeneJET PCR Purification Kit (Thermo). A total of 277 RNA-Seq libraries were made using Illumina TruSeq kits and $2 \times 250$-bp paired-end sequencing was carried out on an Illumina HiSeq 2000 platform at the University of Missouri DNA core. Meanwhile, DNA, used for genome survey sequencing (GSS), was extracted using a Urea buffer method ${ }^{47}$ and $2 \times 150$-bp paired-end libraries were prepared by Illumina TruSeq kits and sequenced using the Illumina NextSeq system at the University of Missouri DNA core.

From each accession, leaf tissue was collected for genome size analyses and phenotype was recorded after flowering. By doing this, we identified 13 putatively $B$. napus accessions which were mislabeled and were actually B. rapa, B. oleracea, or B. juncea. Similarly, one accession identified as B. oleracea was really B. rapa, and three identified as wild $C$ species (2 Brassica cretica and 1 Brassica incana) were actually $B$. oleracea. These cross-species mislabeled samples were discarded. Four B. napus were mislabeled with respect to their subspecies: these accessions were corrected in their identification and used in the study (Supplementary Table 9).

Mapping and SNP calling. All raw paired-end RNA-Seq reads were filtered by Trimmomatic (version 0.36$)^{48}$. Brassica napus reads were both mapped to the B. napus Darmor-bzh genome (version 4.1; http://www.genoscope.cns.fr/brassicanapus/) and rapeseed pan-transcriptome ${ }^{26}$, using TopHat2 (version 2.1.0) ${ }^{49}$ with Bowtie2 (version 2.2.6) ${ }^{50}$. To reduce the influence of mismapping between the $A$ and $C$ sub-genomes due to their high similarity, B. rapa reads were mapped to the A sub-genome of $B$. napus Darmor-bzh genome, while B. oleracea and other wild C species were mapped to the $C$ sub-genome of $B$. napus Darmor-bzh genome using the pipeline described above. Only unique mapped reads were kept for further use. SNPs for each accession were called using HaplotypeCaller in GATK (version 3.7$)^{51}$ as described by the GATK best practices workflow for SNP and indel calling using RNA-Seq data. Finally, we merged different accessions with GenotypeGVCFs in GATK.

We then constructed seven SNP groups for different analysis. Group I (372,546 SNPs): Based on Darmor-bzh genome reference. Combined all B. napus GVCFs and filtered with the following processes: SNPs with a mapping depth $>10$, a mapping quality $>30$ in vcffilter (https://github.com/vcflib/vcflib), and genotyping rate $>50 \%$ and minor allele frequency (MAF) higher than $5 \%$ in PLINK (version $1.9)^{52}$. Group II $(36,829$ SNPs): Based on Darmor- $b z h$ genome reference.

Combined all B. napus GVCFs and filtered with the following processes: SNPs with a mapping depth $>10$, a mapping quality $>30$ in vcffilter (https:/github.com/vcflib/ vcflib), genotyping rate $>90 \%$, minor allele frequency (MAF) higher than $5 \%$ in PLINK (version 1.9) ${ }^{52}$, and only synonymous and non-coding SNPs as annotated by SnpEff (version 4.3) ${ }^{53}$. Group III (24,193 SNPs): Based on Darmor-bzh genome reference. Combined all B. napus, B. rapa, and outgroups GVCFs, SNPs called on A sub-genome and filtered by the same processes as Group II. Group IV $(23,387$ SNPs): Based on Darmor-bzh genome reference. Combined all B. napus, $B$. oleracea, wild C species, and outgroups GVCFs, SNPs called on C sub-genome and filtered by the same processes as Group II. Group V (47,553 SNPs): Based on rapeseed pan-transcriptome reference. Combined all B. napus GVCFs, SNPs then filtered by the same processes as Group II. Group VI (25,569 SNPs): Based on rapeseed pan-transcriptome reference. Combined all B. napus, B. rapa, and outgroups GVCFs, SNPs called on A genome and filtered by the same processes as Group II. Group VII (21,984 SNPs): Based on rapeseed pan-transcriptome reference. Combined all B. napus, B. oleracea, wild $\mathrm{C}$ species, and outgroups GVCFs, SNPs called on C sub-genome and filtered by the same processes as Group II.

To test for SNP ascertainment bias, we counted SNPs in each genetic cluster in each of these four groups based on Darmor-bzh genome. By using worldwide germplasm collections and the GATK best practices pipeline, the SNP numbers and the read-mapping rates of the WEAm genetic cluster, of which the B. napus reference genome belongs to, do not show significantly higher values when compare to the other five genetic clusters in all of the four SNP groups (Supplementary Table 10, Supplementary Fig. 19). This indicates that the SNP ascertainment bias, if present, is small and the SNPs we discovered are good for further downstream analyses ${ }^{54}$. However, our methodology based on RNA-Seq data does provide limited insight into structural rearrangement, which would require genomic structure data.

Population structure and phylogenetic inference. To reduce influences of natural or artificial selection, SNPs only include synonymous and non-coding SNPs, were used for phylogenetic inference and population structure study. Since SNP Group II(36,829 SNPs) and Group V(47,553 SNPs) are obtained based on different reference sequences, they are used to test the reference bias of $B$. napus. The population structure among $B$. napus accessions was inferred by fastSTRUCTURE (version 1.0$)^{27}$ based on a variational Bayesian framework. We tested $K$ from 1 to 10 with five replicates in each $K$ value using default convergence criterions and priors. After that, the best $K$ value was estimated using chooseK.py in the fastSTRUCTURE package and all $K$ values and their replicates were summarized by CLUMPAK $^{55}$. Finally, the multiple $K$ value results of fastSTRUCTURE were reorganized and visualized in Excel corresponding to the accessions order of phylogenetic inference. The maximum-likelihood phylogeny was constructed using RAxML (version 8.2.8) ${ }^{56}$ using the ASC_GTRGAMMA model with 100 bootstrap replicates. Bootstrap support values were then calculated by BOOSTER ${ }^{57}$. Five known closely related species (Brassica nigra, Crambe hispanica, Sinapis alba, Eruca sativa, and Sisymbrium irio) were used as outgroups.

PCA analyses were processed by PLINK (version 1.9) and visualized through Genesis (https://github.com/shaze/genesis). The same SNPs set was also used for Treemix (version 1.13) 28 analyses. To reduce the bias by the recent admixture, only the accessions in these six genetic clusters with their main genetic component larger than 0.6, phylogenetically clustered together, and phenotype matched (Supplementary Data 2) were used in Treemix analyses. The same outgroups used in the phylogenetic analyses were applied here as well. One hundred SNPs were counted as resampling blocks to generate bootstrap replicates. Admixture trees were built with $\mathrm{m}=1-10$ migration events and the model fit for each migration event was evaluated by estimating the proportion of variance explained by each migration model among all the subgroups.

The maximum-likelihood phylogeny among B. napus and one of its ancestors, B. rapa, was constructed using SNP Group III (24,193 SNPs) with the same RAxML parameters and outgroups as above. Additionally, the maximumlikelihood phylogeny among all the $B$. napus, $B$. oleracea, and wild $C$ species was calculated with SNP Group IV (23,387 SNPs) with the same pipeline implemented as for the A sub-genome phylogeny.

Organellar genome assembling and phylogenetic analyses. Three hundred sixteen accessions among these species were also sequenced using genome survey sequencing (GSS) for $2 \times 150$-bp paired-end reads (Supplementary Data 1). Raw reads were filtered by Trimmomatic (version 0.36$)^{48}$ and then mapped using Bowtie2 (version 2.2.6) ${ }^{50}$ to chloroplast and mitochondrial reference genomes, both which contain the chloroplast or mitochondrial genomes of the six species of the Triangle of U (Supplementary Table 11). Next, paired-end reads mapped to 
chloroplast reference genomes were kept and used for de novo assembly of chloroplast genomes for each accession with SPAdes (version 3.11.1) ${ }^{58}$. Scaffolds larger than $300 \mathrm{bp}$ were blasted against the $A$. thaliana chloroplast genes (TAIR, Araport11). The sequences which covered whole $A$. thaliana chloroplast genes (gaps and mismatches in genes were allowed) were defined as homologs of $A$. thaliana genes. Meanwhile, the paired-end reads which mapped to the mitochondrial reference genomes were used for de novo assembly and identification of mitochondrial homologs of $A$. thaliana genes for each accession using the same method.

For chloroplast genes, we discarded accessions which recovered less than 100 homologs of $A$. thaliana genes, resulting in 245 accessions with 92 shared chloroplast genes including 62 genes from single copy regions. For mitochondrial genes, all accessions obtained 45-47 mitochondrial homologs of $A$. thaliana genes, with 42 genes shared by all.

Each of the shared 62 single copy chloroplast genes were aligned with MAFFT (version 7.310) ${ }^{59}$, then cleaned (with parameter: -clean 0.5) and concatenated in Phyutility (version 2.2.6) ${ }^{60}$ for a total alignment length of $29,962 \mathrm{bp}$. Using the same methods, we obtained a 22,937 bp data matrix for mitochondrial sequences. These two concatenated sequences were used to construct two maximumlikelihood phylogenies through RAxML (version 8.2.8) using the GTR-GAMMA substitution model with 100 bootstrap replicates. Bootstrap support values were then calculated using BOOSTER ${ }^{57}$.

Genetic diversity and divergence analysis. SNP Group I (372,546 SNPs) were used to estimate the average nucleotide diversity of each genetic cluster using vcftools (version $0.1 .12 b)^{61}$ with a window size of $100 \mathrm{k} b$ and a step size of $25 \mathrm{k}$ bp. To calculate $\mathrm{F}_{\mathrm{ST}}$ among genetic clusters, we utilized vcftools with a window size of $100 \mathrm{k} \mathrm{bp}$ and a step size of $25 \mathrm{k} \mathrm{bp}$. Linkage disequilibrium $\left(r^{2}\right)$ was calculated by PopLDdecay (version 3.30, https://github.com/BGI-shenzhen/PopLDdecay) with the parameters: -MAF 0.05 -MaxDist 3000 -Het 0.88 -Miss 0.25. All calculations and plots were completed in R (version 3.1.3) and RStudio (version 1.0.136).

Selection analysis. SNP Group I (372,546 SNPs) was phased by Beagle (Version $4.1)$ with GT input format ${ }^{62}$. After that, XP-CLR ${ }^{63}$ and $\pi$ ratio $\left(\pi_{\mathrm{WEAm}} / \pi_{\text {other cluster }}\right)$ methods were used to detect the regions under selective sweeps from the diversification of WEAm to the other genetic clusters. The following parameters were applied to run XP-CLR: -w1 0.00510020001 -p1 0.7. The mean XP-CLR score was calculated using $20 \mathrm{k}$ bp sliding windows with a 10k bp step size. Adjacent $20 \mathrm{k} b \mathrm{bp}$ windows with the top $20 \%$ XP-CLR scores were grouped into a single region, and then merged regions in the top $5 \%$ of the region-wise highest XP-CLR scores were identified, as in Zhang et al. (2017) ${ }^{64}$. To improve prediction accuracy, we also calculated the $\pi$ ratio between WEAm and other genetic clusters with $20 \mathrm{k} b \mathrm{pp}$ sliding windows and a step size of $10 \mathrm{k} \mathrm{bp}$. Windows with the highest $50 \%$ of $\pi$ ratio were merged as single regions. Finally, regions identified by both XP-CLR and $\pi$ ratio were kept as selective sweep regions ${ }^{64}$. On average, $93.36 \%$ of the selective sweep regions identified by XP-CLR were also identified by $\pi$ ratio method (Supplementary Table 12). Finally, figures were made using CIRCOS (version $0.69-4)^{65}$ and $\mathrm{R}$ (version 3.1.3).

Gene expression analysis. After mapping $B$. napus clean reads to the $B$. napus reference genome (version 4.1) and rapeseed pan-transcriptome using TopHat2 (version 2.1.0) with default parameters, the differentially expressed genes were calculated between WEAm and other genetic clusters using the Cufflinks package (version 2.2.1 $)^{66}$. Analyses were run with restrictive conditions of $\mid \log _{2}($ ratio) $\mid>1.0$ and $P$-value $<0.05$. All accessions in the same genetic cluster were treated as replicates. The detailed steps following the Cufflinks RNA-Seq workflow are: Cufflinks to assemble transcripts for each sample; Cuffmerge to construct the final transcriptome; Cuffquant to quantify the mapped reads; and Cuffdiff to calculate the differentially expressed genes.

Functional annotations and metabolic network analyses. The annotations of $B$. napus genes to B. rapa, B. oleracea, and A. thaliana are the same as in Chalhoub et al. (2014) ${ }^{1}$. To identify additional genes of functional importance in B. napus, we aligned coding regions to $B$. rapa, B. oleracea, and $A$. thaliana CDS databases using BLASTN (version 2.4.0) with (-evalue 1E-6) ${ }^{67}$. The gene functions and descriptions were downloaded from www.arabidopsis.org. Overrepresented GO terms associated with unique DEGs in each B. napus genetic cluster were identified using SeqEnrich ${ }^{68}$ base on hypergeometric statistical tests, GO terms in biological processes, molecular functions, and cellular components, with $P$-values $<0.001$ reported.

We have also mapped the B. napus gene differential expression patterns to an $A$. thaliana metabolic network, AraGEM v1.269. The nodes in the network represent biochemical reactions that are catalyzed by enzymes. We calculated a pooled foldchange in expression for each node in the metabolic network: for all the $B$. napus DEGs that are orthologous to $A$. thaliana enzyme-coding genes specific to a node, we took the ratio of the sum FPKM expression in the B. napus genetic clusters (S, SK, or R) over the sum expression in WEAm, and then computed $\log _{2}($ ratio) as the fold-change. The metabolic networks for three genetic clusters were visualized using Gephi (version 0.9.2) ${ }^{70}$. Nodes with expression fold-change $>0$ (on average overexpressed) are colored in red; those that are associated with underexpressed genes are colored in blue. Clustering coefficients for each node were also calculated using Gephi.

Reporting summary. Further information on research design is available in the Nature Research Reporting Summary linked to this article.

\section{Data availability}

Data supporting the findings of this work are available within the paper and its Supplementary Information files. A reporting summary for this Article is available as a Supplementary Information file. The sequencing data for the RNA-Seq and GSS are available at NCBI Sequence Read Archive (SRA) database SRP128554 under project PRJNA428769. This study also used 102 previously published B. rapa transcriptome data sets [SRP072186] for new analyses ${ }^{18}$. The seven SNP groups and the organellar genes are deposited to figshare [https://figshare.com/s/20afelaa9cc682304163]. The source data underlying Figs. 1a and 3e as well as Supplementary Figs. 1, 2, 3, 4, 14, 17 and 18 are provided as a Source Data file. All other data or supporting files are available from the corresponding authors upon request.

Received: 8 October 2018 Accepted: 31 May 2019 Published online: 28 June 2019

\section{References}

1. Chalhoub, B. et al. Early allopolyploid evolution in the post-Neolithic Brassica napus oilseed genome. Science 345, 950-953 (2014).

2. Kimber, D. S. \& McGregor, D. I. (eds.). Brassica oilseeds: production and utilization (National Institute of Agricultural Botany, Cambridge, 1995).

3. Lu, K. et al. Whole-genome resequencing reveals Brassica napus origin and genetic loci involved in its improvement. Nat. Commun. 10, 1154 (2019).

4. Havlickova, L. et al. Validation of an updated Associative Transcriptomics platform for the polyploid crop species Brassica napus by dissection of the genetic architecture of erucic acid and tocopherol isoform variation in seeds. Plant J. 93, 181-192 (2018).

5. USDA. Oilseeds: world markets and trade. https://downloads.usda.library. cornell.edu/usda-esmis/files/tx31qh68h/ng451n501/gt54ks07t/oilseeds.pdf (2018).

6. Iniguez-Luy, F. L. \& Federico, M. L. Genetics and Genomics of the Brassicaceae. (Springer, New York, 2011).

7. Gazave, E. et al. Population genomic analysis reveals differential evolutionary histories and patterns of diversity across subgenomes and subpopulations of Brassica napus L. Front. Plant Sci. 7, 525 (2016).

8. Sturtevant, E. L. Sturtevant's Notes on Edible Plants. (J. B. Lyon Company, State Printers, 1919).

9. Edwards, D., Batley, J., Parkin, I. \& Kole, C. Genetics, Genomics and Breeding of Oilseed Brassicas. (CRC Press, 2011).

10. Tsunoda, S., Hinata, K. \& Gómez-Campo, C. Brassica crops and wild allies. (Japan Scientific Societies Press, Tokyo, 1980).

11. Gómez-Campo, C. \& Prakash, S. in Developments in Plant Genetics and Breeding (ed. Gómez-Campo, C.) 4, 33-58 (Elsevier, Amsterdam, 1999).

12. Song, K. M., Osborn, T. C. \& Williams, P. H. Brassica taxonomy based on nuclear restriction fragment length polymorphisms (RFLPs). Theor. Appl. Genet. 75, 784-794 (1988).

13. Song, K. \& Osborn, T. C. Polyphyletic origins of Brassica napus: new evidence based on organelle and nuclear RFLP analyses. Genome 35, 992-1001 (1992).

14. Rakow, G. in Brassica (ed. Pua, E. C. and Douglas, C. J.) 3-11 (Springer, Berlin, Heidelberg, 2004).

15. Allender, C. J. \& King, G. J. Origins of the amphiploid species Brassica napus L. investigated by chloroplast and nuclear molecular markers. BMC Plant Biol. 10, 54 (2010).

16. Li, P. et al. A phylogenetic analysis of chloroplast genomes elucidates the relationships of the six economically important Brassica species comprising the Triangle of U. Front. Plant Sci. 8, 111 (2017).

17. Palmer, J. D., Shields, C. R., Cohen, D. B. \& Orton, T. J. Chloroplast DNA evolution and the origin of amphidiploid Brassica species. Theor. Appl. Genet. 65, 181-189 (1983).

18. Qi, X. et al. Genomic inferences of domestication events are corroborated by written records in Brassica rapa. Mol. Ecol. 26, 3373-3388 (2017).

19. Becker, H. C., Engqvist, G. M. \& Karlsson, B. Comparison of rapeseed cultivars and resynthesized lines based on allozyme and RFLP markers. Theor. Appl. Genet. 91, 62-67 (1995).

20. Wu, J. et al. Assessing and broadening genetic diversity of a rapeseed germplasm collection. Breed. Sci. 64, 321-330 (2014). 
21. $\mathrm{Wu}, \mathrm{D}$. et al. Whole-genome resequencing of a world-wide collection of rapeseed accessions reveals genetic basis of their ecotype divergence. Mol. Plant. https://doi.org/10.1016/j.molp.2018.11.007 (2018).

22. Xiao, Y. et al. Genetic structure and linkage disequilibrium pattern of a rapeseed (Brassica napus L.) association mapping panel revealed by microsatellites. Theor. Appl. Genet. 125, 437-447 (2012).

23. Delourme, R. et al. High-density SNP-based genetic map development and linkage disequilibrium assessment in Brassica napus L. BMC Genom. 14, 120 (2013).

24. Bus, A., Körber, N., Snowdon, R. J. \& Stich, B. Patterns of molecular variation in a species-wide germplasm set of Brassica napus. Theor. Appl. Genet. 123, 1413-1423 (2011).

25. McVetty, P. B. E. Review of performance and seed production of hybrid Brassicas. in Proc. 9th International Rapeseed Conference, Cambridge 98-103 (GCIRC, 1995)

26. He, Z. et al. Construction of Brassica A and C genome-based ordered pantranscriptomes for use in rapeseed genomic research. Data Brief. 4, 357-362 (2015).

27. Raj, A., Stephens, M. \& Pritchard, J. K. fastSTRUCTURE: variational inference of population structure in large SNP data sets. Genetics 197, 573-589 (2014).

28. Pickrell, J. K. \& Pritchard, J. K. Inference of population splits and mixtures from genome-wide allele frequency data. PLoS Genet 8, e1002967 (2012). between spring and Chinese semi-winter lines. Theor. Appl. Genet. 115, 27-34 (2007).

30. Qian, W. et al. Heterotic patterns in rapeseed (Brassica napus L.): II. Crosses between European winter and Chinese semi-winter lines. Plant Breed. 128, 466-470 (2009).

31. Hurgobin, B. et al. Homoeologous exchange is a major cause of gene presence/ absence variation in the amphidiploid Brassica napus. Plant Biotechnol. J. 16, 1265-1274 (2018).

32. Delourme, R. et al. Characterisation of the radish introgression carrying the $R f o$ restorer gene for the Ogu-INRA cytoplasmic male sterility in rapeseed (Brassica napus L.). Theor. Appl. Genet. 97, 129-134 (1998).

33. Gaeta, R. T., Pires, J. C., Iniguez-Luy, F., Leon, E. \& Osborn, T. C. Genomic changes in resynthesized Brassica napus and their effect on gene expression and phenotype. Plant Cell 19, 3403-3417 (2007).

34. Hansen, L. B., Siegismund, H. R. \& Jørgensen, R. B. Progressive introgression between Brassica napus (oilseed rape) and B. rapa 91, 276-283 (2003).

35. Qian, W. et al. Introgression of genomic components from Chinese Brassica rapa contributes to widening the genetic diversity in rapeseed (B. napus L.), with emphasis on the evolution of Chinese rapeseed. Theor. Appl. Genet. 113, 49-54 (2006)

36. Liu, S. et al. The Brassica oleracea genome reveals the asymmetrical evolution of polyploid genomes. Nat. Commun. 5, 3930 (2014).

37. Fang, L., Guan, X. \& Zhang, T. Asymmetric evolution and domestication in allotetraploid cotton (Gossypium hirsutum L.). Crop J. 5, 159-165 (2017).

38. Wei, D. et al. A genome-wide survey with different rapeseed ecotypes uncovers footprints of domestication and breeding. J. Exp. Bot. 68, 4791-4801 (2017).

39. Mason, A. S. et al. Agricultural selection and presence-absence variation in spring-type canola germplasm. Crop Pasture Sci. 69, 55-64 (2018).

40. Zhao, X. et al. Breeding signature of combining ability improvement revealed by a genomic variation map from recurrent selection population in Brassica napus. Sci. Rep. 6, 29553 (2016).

41. Cheng, F. et al. Subgenome parallel selection is associated with morphotype diversification and convergent crop domestication in Brassica rapa and Brassica oleracea. Nat. Genet. 48, 1218-1224 (2016).

42. Schiessl, S., Samans, B., Hüttel, B., Reinhard, R. \& Snowdon, R. J. Capturing sequence variation among flowering-time regulatory gene homologs in the allopolyploid crop species Brassica napus. Front. Plant Sci. 5, 404 (2014).

43. Schiessl, S., Huettel, B., Kuehn, D., Reinhardt, R. \& Snowdon, R. Postpolyploidisation morphotype diversification associates with gene copy number variation. Sci. Rep. 7, 41845 (2017).

44. Zhou, Z. et al. Resequencing 302 wild and cultivated accessions identifies genes related to domestication and improvement in soybean. Nat. Biotechnol. 33, 408-414 (2015)

45. Wing, R. A., Purugganan, M. D. \& Zhang, Q. The rice genome revolution: from an ancient grain to Green Super Rice. Nat. Rev. Genet. https://doi.org/ 10.1038/s41576-018-0024-z (2018).

46. Zsögön, A. et al. De novo domestication of wild tomato using genome editing. Nat. Biotechnol. https://doi.org/10.1038/nbt.4272 (2018).

47. Leach, K. A. \& McSteen, P. C. Genomic DNA isolation from maize (Zea mays) leaves using a simple, high-throughput protocol. Curr. Protoc. Plant. https:// doi.org/10.1002/cppb.20000 (2016).
48. Bolger, A. M., Lohse, M. \& Usadel, B. Trimmomatic: a flexible trimmer for Illumina sequence data. Bioinformatics 30, 2114-2120 (2014).

49. Kim, D. et al. TopHat2: accurate alignment of transcriptomes in the presence of insertions, deletions and gene fusions. Genome Biol. 14, R36 (2013).

50. Langmead, B. \& Salzberg, S. L. Fast gapped-read alignment with Bowtie 2. Nat Methods 9, 357-359 (2012).

51. McKenna, A. et al. The Genome Analysis Toolkit: a MapReduce framework for analyzing next-generation DNA sequencing data. Genome Res. 20, 1297-1303 (2010)

52. Purcell, S. et al. PLINK: a tool set for whole-genome association and population-based linkage analyses. Am. J. Hum. Genet. 81, 559-575 (2007).

53. Cingolani, P. et al. A program for annotating and predicting the effects of single nucleotide polymorphisms, SnpEff: SNPs in the genome of Drosophila melanogaster strain w1118. iso-2; iso-3 6, 80-92 (2012).

54. Lachance, J. \& Tishkoff, S. A. SNP ascertainment bias in population genetic analyses: why it is important, and how to correct it. Bioessays 35, 780-786 (2013).

55. Kopelman, N. M., Mayzel, J., Jakobsson, M., Rosenberg, N. A. \& Mayrose I. Clumpak: a program for identifying clustering modes and packaging population structure inferences across K. Mol. Ecol. Resour. 15, 1179-1191 (2015).

56. Stamatakis, A. RAxML version 8: a tool for phylogenetic analysis and post-analysis of large phylogenies. Bioinformatics 30, 1312-1313 (2014).

57. Lemoine, F. et al. Renewing Felsenstein's phylogenetic bootstrap in the era of big data. Nature 556, 452-456 (2018)

58. Bankevich, A. et al. SPAdes: a new genome assembly algorithm and its applications to single-cell sequencing. J. Comput. Biol. 19, 455-477 (2012).

59. Katoh, K. \& Standley, D. M. MAFFT multiple sequence alignment software version 7: improvements in performance and usability. Mol. Biol. Evol. 30, 772-780 (2013)

60. Smith, S. A. \& Dunn, C. W. Phyutility: a phyloinformatics tool for trees, alignments and molecular data. Bioinformatics 24, 715-716 (2008).

61. Danecek, P. et al. The variant call format and VCFtools. Bioinformatics 27, 2156-2158 (2011).

62. Browning, S. R. \& Browning, B. L. Rapid and accurate haplotype phasing and missing-data inference for whole-genome association studies by use of localized haplotype clustering. Am. J. Hum. Genet. 81, 1084-1097 (2007).

63. Chen, H., Patterson, N. \& Reich, D. Population differentiation as a test for selective sweeps. Genome Res. 20, 393-402 (2010).

64. Zhang, L. et al. RNA sequencing provides insights into the evolution of lettuce and the regulation of flavonoid biosynthesis. Nat. Commun. 8, 2264 (2017).

65. Krzywinski, M. et al. Circos: an information aesthetic for comparative genomics. Genome Res. 19, 1639-1645 (2009).

66. Ghosh, S. \& Chan, C.-K. K. Analysis of RNA-Seq data using TopHat and Cufflinks. Methods Mol. Biol. 1374, 339-361 (2016).

67. Camacho, C. et al. BLAST+: architecture and applications. BMC Bioinforma. 10, 421 (2009).

68. Becker, M. G., Walker, P. L., Pulgar-Vidal, N. C. \& Belmonte, M. F. SeqEnrich: a tool to predict transcription factor networks from co-expressed Arabidopsis and Brassica napus gene sets. PLoS ONE 12, e0178256 (2017).

69. Bekaert, M., Edger, P. P., Pires, J. C. \& Conant, G. C. Two-phase resolution of polyploidy in the Arabidopsis metabolic network gives rise to relative and absolute dosage constraints. Plant Cell 23, 1719-1728 (2011).

70. Bastian, M., Heymann, S. \& Jacomy, M. Gephi: an open source software for exploring and manipulating networks. in Third International AAAI Conference on Weblogs and Social Media. (AAAI Publications, 2009)

\section{Acknowledgements}

We thank Jun Zou for sharing the Brassica napus genetic map with us. We would like to thank Sarah Unruh, Sarah Turner, Paul Blischak, and Andrea Ravelo for their assistance with this study. H.A, X.Q, M.L.G, Y.H., S.C.G, M.E.M, M.S.B, G.C.C. and J.C.P were supported by a National Science Foundation (IOS-1339156). H.A., B.Y. and T.F. were supported by the Fundamental Research Funds for the Central Universities (2662016PY063).

\section{Author contributions}

J.C.P., B.Y., H.A., M.S.B. and T.F. designed the experiments. H.A., X.Q., Y.H. and G.C.C analyzed the data, H.A., M.L.G. and S.C.G. prepared RNA and DNA for sequencing, H. A., S.C.G. and M.E.M. planted, sampled, and phenotyped the plant materials. G.T. and A C.M. provide part of plant materials and comments. H.A., M.L.G., M.E.M., and J.C.P. wrote the manuscript. 


\section{Additional information}

Supplementary Information accompanies this paper at https://doi.org/10.1038/s41467019-10757-1.

Competing interests: The authors declare no competing interests.

Reprints and permission information is available online at http://npg.nature.com/ reprintsandpermissions/

Journal peer review information: Nature Communications thanks the anonymous reviewers for their contribution to the peer review of this work.

Publisher's note: Springer Nature remains neutral with regard to jurisdictional claims in published maps and institutional affiliations. (c) (i) Open Access This article is licensed under a Creative Commons Attribution 4.0 International License, which permits use, sharing, adaptation, distribution and reproduction in any medium or format, as long as you give appropriate credit to the original author(s) and the source, provide a link to the Creative Commons license, and indicate if changes were made. The images or other third party material in this article are included in the article's Creative Commons license, unless indicated otherwise in a credit line to the material. If material is not included in the article's Creative Commons license and your intended use is not permitted by statutory regulation or exceeds the permitted use, you will need to obtain permission directly from the copyright holder. To view a copy of this license, visit http://creativecommons.org/ licenses/by/4.0/.

(C) The Author(s) 2019 